Louisiana State University

LSU Digital Commons

Faculty Publications

Department of Physics \& Astronomy

$1-1-2013$

\title{
Sensitive estimation of angular displacements using weak measurements
}

Omar S. Magaña-Loaiza

University of Rochester Institute of Optics

Mohammad Mirhosseini

University of Rochester Institute of Optics

Brandon Rodenburg

University of Rochester Institute of Optics

Robert W. Boyd

University of Rochester Institute of Optics

Follow this and additional works at: https://digitalcommons.Isu.edu/physics_astronomy_pubs

\section{Recommended Citation}

Magaña-Loaiza, O., Mirhosseini, M., Rodenburg, B., \& Boyd, R. (2013). Sensitive estimation of angular displacements using weak measurements. Optics InfoBase Conference Papers https://doi.org/10.1364/ cqo.2013.m6.45

This Conference Proceeding is brought to you for free and open access by the Department of Physics \& Astronomy at LSU Digital Commons. It has been accepted for inclusion in Faculty Publications by an authorized administrator of LSU Digital Commons. For more information, please contact ir@lsu.edu. 


\title{
Sensitive estimation of angular displacements using weak measurements
}

\author{
Omar S. Magaña-Loaiza ${ }^{1}$, Mohammad Mirhosseini ${ }^{1}$, Brandon Rodenburg $^{1}$ and Robert $^{-}$ \\ W. Boyd ${ }^{1,2}$ \\ ${ }^{1}$ The Institute of Optics, University of Rochester, Rochester, New York 14627 USA \\ ${ }^{2}$ Department of Physics, University of Ottawa, Ottawa, ON K1N 6N5 Canada \\ omarmaganaloaiza@rochester.edu
}

\begin{abstract}
We demonstrate an experimental method that allows for sensitive measurements of angular position of light using weak value amplification. This offers an alternative to previously established methods that use nonclassical light for angular displacement estimation.
\end{abstract}

(C) 2013 Optical Society of America

OCIS codes: $270.5585,270.1670,270.5570$.

We use an interferometric arrangement to amplify the resolution and sensitivity of angular-displacement measurements. The sensitivity of our measurement scheme is due to the amplification of the weak values of the angular position. This is achieved by coupling the angular displacement of the photons with its polarization [1].

\section{Amplification of Angular Displacement}

The amplification of angular displacement is carried out in the first part of our experiment (Fig. 1). We select an initial polarization state using a half wave plate and a polarizer, which is split into two paths by a polarizing beam splitter. We prepare two angular position states by impressing spatial and phase information onto the beams, by using two SLMs. The angular states, with an angular width $\beta$, are spatially separated by an angular displacement $\Delta \phi$, which is small relative to $\beta$. This constitutes our weak measurement. The two beams are then recombined by a second beam splitter. Finally a strong measurement or post-selection is performed using a second polarizer. By careful choice of the polarization angles used in the pre and post selected states, the angular displacement $\Delta \phi$ becomes amplified.

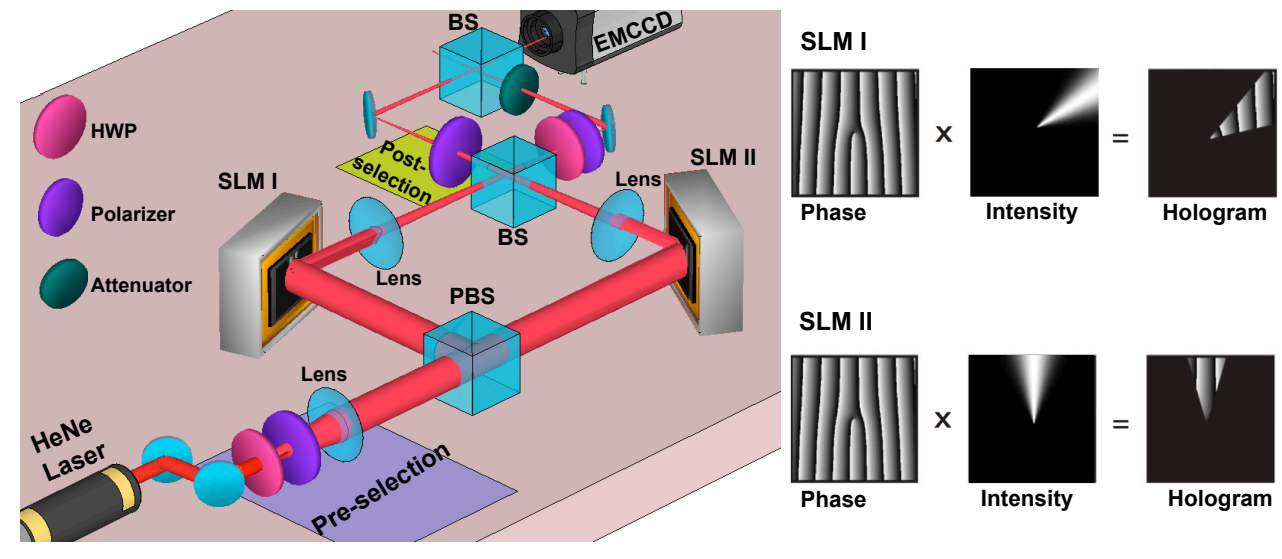

Fig. 1. Schematic of the experimental setup and phase holograms impressed on the SLMs.

We must couple the desired observable of the measured system to a pointer. For our setup we coupled the polarization with orbital angular momentum, $\hat{\ell}$ (the generator of angular rotations). This gives us the following interaction Hamiltonian:

$$
H_{i}=g \hat{\ell} \hat{S}
$$


where $\hat{S}=|H\rangle\langle H|$ and $g=\Delta \phi$ is the coupling constant. This coupling shifts the position of the pointer by an amount proportional to the weak value of $\hat{S}$. The initial state is given by:

$$
\left.\left.\left|\psi^{i}\right\rangle=\mid \text { spin }\right\rangle \mid \text { spatial }\right\rangle=|D\rangle\left|\psi_{T}\right\rangle
$$

where $|D\rangle$ represents the original prepared polarization, in this case diagonal, and $\left|\psi_{T}\right\rangle$ indicates the superposition of the angular states, with azimuthal gaussian intensity distribution, given by the two slits.

The expectation value of the angular position in the post-selected state, $|A+\delta\rangle$, rotates the superposition of the two angular states, the rotation is given by $\Delta\langle\phi\rangle_{r} \approx \Delta \phi S_{w}$, where the amplification is determined by the weak value $S_{w}=\frac{\langle A+\delta|\hat{S}| D\rangle}{\langle A+\delta|| D\rangle}$. The amplification depends on the original and post-selected angles, as well as the strength of the measurement. This fact, allows us to perform an exact simulation of the experiment. We have considered a situation where the angular displacement between the two slits (one with polarization $\mathrm{H}$ and the other with $\mathrm{V}$ ) is pi/8000, and we have amplified the angular displacement 366.27 times by post-selecting.

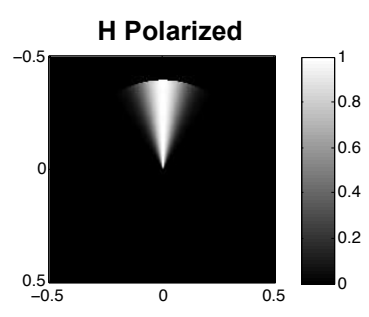

a)

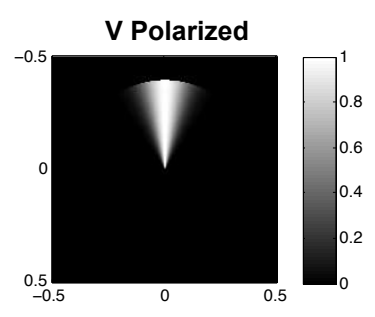

b)

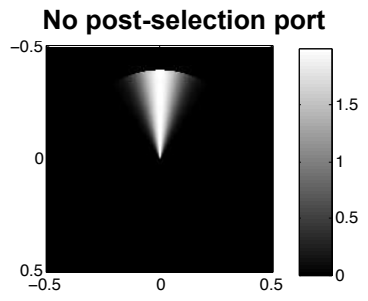

c)

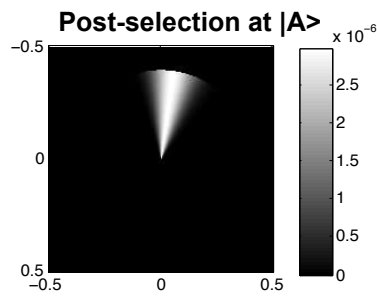

d)

Fig. 2. Simulation of the experiment; a) the angular state with $\mathrm{H}$ polarization is sent to one of the arms, while the angular state with V polarization is sent to the other arm b), one is displaced pi/8000 radians with respect to the other. The superposition of these states, generate the state in c). The post selection shows an amplification of the angular displacement by a factor of $366.27 \mathrm{~d}$ ).

Fig. 2 c) shows the output state in the output ports of the second beam splitter, meanwhile Fig. 2 d) shows the state after post-selection.

\section{Enhanced Resolution and Sensitivity without Entanglement}

The second part of our experiment consists of the interference between the state post-selected at $|A+\delta\rangle$ and the state with no post-selection, which is $|D\rangle$. The beam with $\mathrm{D}$ polarization is stronger than the beam with A polarization, therefore attenuation and polarization rotation is needed. This lead us to the following interference pattern in the angular momentum domain:

$$
I=\frac{\beta}{2 \pi} \operatorname{Sinc}^{2}\left(\frac{\ell \beta}{2}\right)\left(1+\operatorname{Cos}\left(\ell \Delta \phi S_{w}\right)\right)
$$

It is easy to notice that the resolution of the fringes has been increased by a factor proportional to $S_{w}$. As shown in Fig. 1, we chose to measure the interference pattern given by Eq. (3) by projecting the field into a mode with OAM $\ell$. This procedure offers an alternative, without requiring entanglement, to existing methods for estimating angular displacements [2]. In our case, due to the amplification of the weak value, the sensitivity is increased by a factor of $S_{w}$. This effect is similar to the one achieved with N00N states, but it is important to notice that it is easier to create a high value for $S_{w}$ than high N00N states.

\section{References}

1. N. W. M. Ritchie, J. G. Story, and Randall G. Hulet, "Realization of a measurement of a weak value," Phys. Rev. Lett. 66, 11071110 (1991).

2. A. K. Jha, G. S. Agarwal, and R. W. Boyd, "Supersensitive measurement of angular displacements using entangled photons,” Phys. Rev. A 84, 063847 (2011). 